\title{
The interplay of microbiota and hormone regulation in men with prostate cancer
}

\author{
Mark C. Markowski ${ }^{1} \cdot$ Karen S. Sfanos $\mathbb{1}^{1,2,3}$
}

Received: 29 March 2021 / Revised: 15 April 2021 / Accepted: 28 April 2021 / Published online: 18 May 2021

(c) The Author(s), under exclusive licence to Springer Nature Limited 2021

The interplay between the gastrointestinal (GI) microbiota and drug metabolism and/or efficacy is an emergent area of interest in clinical oncology. In men with prostate cancer, the GI microbiota may specifically influence hormonal therapies, and vice versa. A study by Li et al. published in this issue of Prostate Cancer and Prostatic Diseases examined the GI microbiota of men undergoing treatment with androgen deprivation therapy (ADT) versus radical prostatectomy. Compared to the radical prostatectomy group, men undergoing treatment with ADT had (1) lower GI microbial diversity and Firmicutes-to-Bacteroidetes ratio (used as an indicator of GI health) and (2) enriched predicted prevalence of the biosynthesis of lipopolysaccharide (endotoxin) and propanoate. The authors hypothesize that the compositional and functional differences observed in men undergoing ADT may contribute to the known development of metabolic complications from ADT, but caution that longitudinal studies to both confirm that GI microbiota profiles change upon ADT treatment as well as explore their relationship to the development of metabolic complications are needed [1].

The study by $\mathrm{Li}$ et al. [1] builds on previous work demonstrating that commonly used nonantibiotic drugs, including proton pump inhibitors (PPIs), metformin, antidepressants, and oral steroids impact the composition and metabolic function of the GI microbiota [2]. In some instances, these changes in microbial composition had unexpected implications for overall health. For example, the

Karen S. Sfanos

ksfanos@jhmi.edu

1 Sidney Kimmel Comprehensive Cancer Center, Baltimore, MD, USA

2 Department of Pathology, Johns Hopkins University School of Medicine, Baltimore, MD, USA

3 Department of Urology, James Buchanan Brady Urological Institute, Johns Hopkins University School of Medicine, Baltimore, MD, USA authors quantified carriage of antibiotic resistance genes in participants using PPIs and report consistent increases in antibiotic resistance gene markers in PPI users compared to nonusers. In regards to use of oral steroids, the authors noted an increase in the prevalence of Methanobrevibacter smithii in users of glucocorticoids including prednisolone, prednisone, hydrocortisone, and cortisone. Interestingly, M. smithii is a methanogen implicated in the development of obesity via its capacity to increase caloric harvest [3]. The authors hypothesize that an increased abundance of $M$. smithii in participants taking oral steroids could potentially explain the weight gain associated with oral steroid use.

Previous studies in animal models also demonstrate that the GI microbiota can influence circulating hormone levels [4] and be altered by castration [5]. Likewise, a study in men with prostate cancer reported measurable compositional differences in the GI microbiota of men taking oral antiandrogens compared to men with and without prostate cancer who were not undergoing treatment with these therapies [6]. Species overrepresented in men taking oral antiandrogens included species previously linked to response to anti-PD-1 immunotherapy such as Akkermansia muciniphila [7] and Ruminococcaceae spp [8], and functional analyses indicated an enriched representation of bacterial gene pathways involved in steroid biosynthesis and steroid hormone biosynthesis. We speculate that alterations to the GI microbiota by oral hormonal therapies for prostate cancer may influence clinical response to oral antiandrogens or future therapies including immunotherapy. This notion is supported in a study by Daisley et al. who demonstrated the depletion of Corynebacterium spp. in men on ADT alone and an increase in the health-associated Akkermansia muciniphila upon treatment with ADT and abiraterone acetate [9]. These authors further report a coinciding predicted increase in bacterial biosynthesis of vitamin $\mathrm{K} 2$ in correlation to increases in A. muciniphila, which may affect the efficacy of abiraterone acetate.

Collectively, the studies in prostate cancer to date demonstrate an impact of hormonal therapies on the 
composition of the GI microbiota, and suggest that these compositional changes may impact response to therapy and/ or mediate therapy-related comorbidities.

\section{Compliance with ethical standards}

Conflict of interest The authors declare no competing interests.

Publisher's note Springer Nature remains neutral with regard to jurisdictional claims in published maps and institutional affiliations.

\section{References}

1. Li J, Wang L, Wong C, Chiu P, Teoh J, Kwok H, et al. A cross sectional study on gut microbiota in prostate cancer patients with prostatectomy or androgen deprivation therapy. Prostate Cancer Prostatic Dis. 2021.

2. Vich Vila A, Collij V, Sanna S, Sinha T, Imhann F, Bourgonje AR, et al. Impact of commonly used drugs on the composition and metabolic function of the gut microbiota. Nat Commun. 2020;11:362.

3. Mathur R, Kim G, Morales W, Sung J, Rooks E, Pokkunuri V, et al. Intestinal methanobrevibacter smithii but not total bacteria is related to diet-induced weight gain in rats. Obesity. 2013;21:748-54.

4. Markle JGM, Frank DN, Mortin-Toth S, Robertson CE, Feazel LM, Rolle-Kampczyk U, et al. Sex differences in the gut microbiome drive hormone-dependent regulation of autoimmunity. Science. 2013;339:1084-8.

5. Harada N, Hanaoka R, Horiuchi H, Kitakaze T, Mitani T, Inui H, et al. Castration influences intestinal microflora and induces abdominal obesity in high-fat diet-fed mice. Sci Rep. 2016;6:23001.

6. Sfanos KS, Markowski MC, Peiffer LB, Ernst SE, White JR, Pienta $\mathrm{KJ}$, et al. Compositional differences in gastrointestinal microbiota in prostate cancer patients treated with androgen axis-targeted therapies. Prostate Cancer Prostatic Dis. 2018;21:539-48.

7. Routy B, Le Chatelier E, Derosa L, Duong CPM, Alou MT, Daillère $\mathrm{R}$, et al. Gut microbiome influences efficacy of PD1-based immunotherapy against epithelial tumors. Science. 2018;359:91-7.

8. Gopalakrishnan V, Spencer CN, Nezi L, Reuben A, Andrews MC, Karpinets TV, et al. Gut microbiome modulates response to antiPD-1 immunotherapy in melanoma patients. Science. 2018;359:97-103.

9. Daisley BA, Chanyi RM, Abdur-Rashid K, Al KF, Gibbons S, Chmiel JA, et al. Abiraterone acetate preferentially enriches for the gut commensal Akkermansia muciniphila in castrate-resistant prostate cancer patients. Nat Commun. 2020;11:4822. 